\title{
Genetic evidence of a causal effect of insulin resistance on branched-chain amino acid levels
}

\author{
Yuvaraj Mahendran $^{1,2}$ • Anna Jonsson ${ }^{1}$ - Christian T. Have ${ }^{1} \cdot$ Kristine H. Allin $^{1}$ • \\ Daniel R. Witte ${ }^{2,3}$ - Marit E. Jørgensen ${ }^{4} \cdot$ Niels Grarup ${ }^{1}$ • Oluf Pedersen ${ }^{1}$ • \\ Tuomas O. Kilpeläinen ${ }^{1}$ • Torben Hansen ${ }^{1}$
}

Received: 5 October 2016 / Accepted: 25 January 2017 /Published online: 10 February 2017

(C) Springer-Verlag Berlin Heidelberg 2017

\begin{abstract}
Aims/hypothesis Fasting plasma levels of branched-chain amino acids (BCAAs) are associated with insulin resistance, but it remains unclear whether there is a causal relation between the two. We aimed to disentangle the causal relations by performing a Mendelian randomisation study using genetic variants associated with circulating BCAA levels and insulin resistance as instrumental variables.

Methods We measured circulating BCAA levels in blood plasma by NMR spectroscopy in 1,321 individuals from the ADDITION-PRO cohort. We complemented our analyses by using previously published genome-wide association study (GWAS) results from the Meta-Analyses of Glucose and Insulin-related traits Consortium (MAGIC) $(n=46,186)$ and from a GWAS of serum BCAA levels $(n=24,925)$. We used a genetic risk score (GRS), calculated using ten established fasting serum insulin associated variants, as an instrumental variable for insulin resistance. A GRS of three variants increasing circulating BCAA levels was used as an instrumental variable for circulating BCAA levels.
\end{abstract}

Electronic supplementary material The online version of this article (doi:10.1007/s00125-017-4222-6) contains peer-reviewed but unedited supplementary material, which is available to authorised users.

Torben Hansen

torben.hansen@sund.ku.dk

1 Novo Nordisk Foundation Center for Basic Metabolic Research, Section of Metabolic Genetics, Faculty of Health and Medical Sciences, University of Copenhagen, Universitetsparken 1, 2100 Copenhagen, Denmark

2 The Danish Diabetes Academy, Odense, Denmark

3 Institute of Public Health, University of Aarhus, Aarhus, Denmark

4 Steno Diabetes Center, Gentofte, Denmark
Results Fasting plasma BCAA levels were associated with higher HOMA-IR in ADDITION-PRO ( $\beta 0.137$ [95\% CI $0.08,0.19] p=6 \times 10^{-7}$ ). However, the GRS for circulating BCAA levels was not associated with fasting insulin levels or HOMA-IR in ADDITION-PRO $(\beta-0.011$ [95\% CI -0.053 , $0.032] p=0.6$ and $\beta-0.011$ [95\% CI $-0.054,0.031] p=0.6$, respectively) or in GWAS results for HOMA-IR from MAGIC ( $\beta$ for valine-increasing GRS -0.012 [95\% CI $-0.069,0.045]$ $p=0.7)$. By contrast, the insulin-resistance-increasing GRS was significantly associated with increased BCAA levels in ADDITION-PRO ( $\beta 0.027$ [95\% CI 0.005, 0.048] $p=0.01)$ and in GWAS results for serum BCAA levels $(\beta 1.22$ [95\% CI $0.71,1.73] p=4 \times 10^{-6}, \beta 0.96$ [95\% CI $\left.0.45,1.47\right]$ $p=3 \times 10^{-4}$, and $\beta 0.67$ [95\% CI $\left.0.16,1.18\right] p=0.01$ for isoleucine, leucine and valine levels, respectively) and instrumental variable analyses in ADDITION-PRO indicated that HOMA-IR is causally related to higher circulating fasting BCAA levels ( $\beta 0.73$ [95\% CI 0.26, 1.19] $p=0.002$ ).

Conclusions/interpretation Our results suggest that higher BCAA levels do not have a causal effect on insulin resistance while increased insulin resistance drives higher circulating fasting BCAA levels.

Keywords Branched-chain amino acids $\cdot$ Fasting insulin variants $\cdot$ Genetic risk score $\cdot$ Insulin resistance $\cdot$ Mendelian randomisation
Abbreviations
BCAA Branched-chain amino acid
GRS
Genetic risk score
GWAS Genome-wide association study
MAGIC The Meta-Analyses of Glucose and Insulin-related traits Consortium
SNP Single nucleotide polymorphism 


\section{Introduction}

Insulin resistance is one of the primary and earliest detectable defects of metabolic dysregulation that leads to type 2 diabetes [1]. Multiple epidemiological studies have shown an association between increased fasting levels of circulating branched-chain amino acids (BCAAs) and increased insulin resistance [2]. A recent study provided a potential mechanistic explanation for how higher BCAA levels could cause insulin resistance by promoting lipid accumulation in the muscle through 3-hydroxyisobutyrate, a catabolic intermediate of the BCAA valine [3]. However, other studies indicate that increased circulating BCAA levels may be the consequence of impaired insulin action and merely a marker of increased insulin resistance [2].

Genetic variants identified in genome-wide association studies (GWAS) for circulating BCAA levels and insulin resistance can be utilised as instrumental variables in Mendelian randomisation [4-6], to examine causal relations between the two. So far, four variants have been shown to associate with circulating BCAA levels in the fasting state [5]. The rs9637599 variant associated with circulating BCAA levels is located $2 \mathrm{~kb}$ upstream of the PPM1K gene, which encodes a mitochondrial phosphatase known to play a vital role in BCAA metabolism [7]. Three other known variants for circulating BCAA levels are rs10211524, located near to the SLC1A4 gene (encoding solute carrier family 1, member 4), rs7406661 near the ASGR1 gene (encoding asialoglycoprotein receptor 1 ), and the missense variant rs 1260326 in the GCKR gene (encoding glucokinase receptor). GWAS for fasting insulin levels have uncovered 24 loci, of which ten have been robustly associated with insulin resistance $[4,6]$.

In the present study, we used genetic risk scores (GRSs) of the known variants associated with circulating BCAA levels or insulin resistance as instrumental variables to examine the causal effect of fasting plasma BCAA levels on insulin resistance and vice versa.

\section{Methods}

Study population ADDITION-PRO is a longitudinal cohort study of individuals at low to high risk of type 2 diabetes, nested within the population-based ADDITION-Denmark trial of screen-detected diabetes [8,9]. A total of 1,321 individuals were included in the present study, 1182 individuals were included as high-risk individuals (150 had isolated impaired fasting glucose [IFG]; 133 had isolated impaired glucose tolerance [IGT]; 50 had both IFG and IGT; 849 had high diabetes risk) and 139 were classed as having low diabetes risk. High ( $\geq 5$ points on diabetes risk score) and low ( $<5$ points) diabetes risk were assessed using a slightly modified version of the Danish diabetes risk questionnaire, completed by study participants. The questionnaire scored the participants on the basis of their levels of known risk factors for diabetes including age, sex, BMI, known hypertension, family history of type 2 diabetes, gestational diabetes and leisure time physical activity [8]. Characteristics of the study participants are given in electronic supplementary material (ESM) Table 1. All individuals who fasted $\leq 7 \mathrm{~h}$ before fasting blood samples were taken, or who used diabetes medication, were excluded. The study was approved by the Ethics Committee of the Central Denmark Region (journal no. 20080229) and was conducted in accordance with the Helsinki Declaration. All participants provided written informed consent.

Anthropometric measurements Body weight, height, and hip and waist circumference were measured as previously described [10]. BMI was calculated as weight $(\mathrm{kg})$ divided by height (m) squared.

Measurement of insulin resistance Fasting plasma glucose and serum insulin measurements have been described previously [10]. HOMA-IR was calculated as fasting glucose $(\mathrm{mmol} / \mathrm{l}) \times$ [fasting insulin $(\mathrm{pmol} / \mathrm{l}) / 6.945] / 22.5$ [10] .

Measurement of fasting plasma BCAAs Fasting blood samples were collected in EDTA tubes and plasma was stored at $-80^{\circ} \mathrm{C}$. Proton NMR spectroscopy was used to measure amino acid levels. NMR methods have been described in detail previously [5]. Total circulating BCAA levels were defined as the sum of fasting isoleucine, leucine and valine levels.

Genotyping We genotyped 1,657 participants from the ADDITION-PRO cohort using the Illumina Infinium HumanCoreExome Beadchip (Illumina, San Diego, CA, USA). Genotypes were called using the Genotyping module (version 1.9.4) of GenomeStudio software (version 2011.1, Illumina). We excluded individuals who were first degree relatives, duplicates, outliers (ethnic or otherwise) as determined by visual inspection of principal components, or had extreme inbreeding coefficients (by visual inspection of $F$-statistics), mislabelled sex, or call rate $<95 \%$, leaving 1,348 individuals who passed quality control criteria. A total of 1,321 individuals had information on total circulating BCAA levels, HOMA-IR and fasting serum insulin levels. The quality control of genotype data was based on the guidelines described previously [11]. The genotypes used in the current study were imputed with high quality (IMPUTE2 info $>0.95$ ) into the 1000 genomes phase 1 panel using IMPUTE2 [12, 13]. The imputation quality was high (IMPUTE2 info $>0.95$ ) for all imputed variants included in the current study (four of ten fasting insulin variants 
[rs4846565, rs3822072, rs6822892, rs4865796] and three variants for circulating BCAA levels [rs9637599, rs10211524, rs7406661]). All variants included in this study were in Hardy-Weinberg equilibrium $(p>0.05)$.

Instrumental variables To generate an instrumental variable for circulating BCAA levels, we used publicly available GWAS meta-analysis results for isoleucine, leucine and valine levels from up to 24,900 individuals [5]. We calculated an unweighted GRS using the most significant variant associated with circulating BCAA levels in three of the four known BCAA loci (ESM Table 2). We excluded one known locus, in $G C K R$, owing to its known pleotropic effect on multiple human complex traits [14]. In addition, we constructed a GRS for circulating BCAA levels with and without the PPMK1 single nucleotide polymorphism (SNP) rs9637599 and performed analysis considering the association of rs9637599 with BMI $(p=0.035)[15]$ and obesity class I $(p=0.00042)$ [16] in the publicly available GWAS summary data.

To generate an instrumental variable for insulin resistance, we calculated an unweighted GRS using the fasting insulin associated variants reported in a GWAS meta-analysis of the Meta-Analyses of Glucose and Insulin-related traits Consortium (MAGIC) [4] (ESM Table 3). To improve the specificity of the score, we only included ten fasting serum insulin variants known to be associated with lower HDL-cholesterol and higher triacylglycerol, a biochemical hallmark of insulin resistance [6]. The score was constructed by summing the number of insulin-increasing alleles reported by MAGIC [4].

We complemented our analyses of individual-level data in ADDITION-PRO by testing the associations of valine, leucine and isoleucine GRSs with HOMA-IR using GWAS summary results from MAGIC [4]. Similarly, we tested for the association of fasting insulin GRS with valine, leucine, and isoleucine levels using GWAS summary results by Kettunen et al [5]. We implemented a multi-SNP genotype risk score method (R package 'gtx') used previously by Dastani et al [17]. This method approximates the average effect of variants that increase circulating BCAA levels on insulin resistance, and that of insulin-resistance-increasing variants on plasma BCAA levels, in publicly available GWAS meta-analysis results, enabling the estimation of causal effects in large sample sizes $[17,18]$. However, the method does not involve a formal instrumental variable analysis. In brief, we identified three SNPs (rs10211524, rs9637599, rs7406661) that were statistically or nominally associated with valine levels, leucine levels, or isoleucine levels (ESM Table 2), to test for the association of valine GRS, leucine GRS or isoleucine GRS with HOMA-IR in GWAS summary results from MAGIC. We removed the variant rs7406661 from the analyses because this SNP was not available in GWAS summary results for HOMA-IR from MAGIC.
Statistical analysis Statistical analyses were performed using R, version 3.1.3 (www.R-project.org/). We removed extreme outliers for circulating BCAA levels ( $>4 \mathrm{SD}$ ), and normalised the distributions of circulating BCAA levels, HOMA-IR and insulin using rank-based inverse normal transformation. Multiple linear regression analyses were used to test for associations between circulating BCAAs, insulin resistance and genetic variants adjusting for age, sex and BMI. We performed a bi-directional Mendelian randomisation analysis using two-stage least-squares regression to estimate the possible causal relationship between plasma BCAA levels and insulin resistance, and vice versa [19].

\section{Results}

Higher fasting plasma BCAA levels were significantly associated with increased fasting serum insulin levels ( $\beta 0.127$ [95\% CI 0.073, 0.181] $\left.p=4 \times 10^{-6}\right)$ and HOMA-IR ( $\beta 0.137[95 \%$ CI $0.083,0.190] p=6 \times 10^{-7}$ ) in the ADDITION-PRO cohort (Fig. 1). The GRS for circulating BCAA levels increased plasma BCAA levels by 0.045 SD/allele ([95\% CI 0.002, 0.087] $p=0.04, n=1,321)$ but no association was found between the BCAA GRS and fasting insulin levels $(\beta=-0.011 \mathrm{SD} /$ allele, [95\% CI $-0.053,0.032]$ $p=0.6)$ or HOMA-IR $(\beta=-0.011 \mathrm{SD} /$ allele, $[95 \% \mathrm{CI}-0.054$, $0.031] p=0.6$ ) (Table 1). Exclusion of individuals belonging to the low diabetes risk group of ADDITION-PRO $(n=139)$ did not change the results (circulating BCAA levels with HOMA-IR, $\beta=0.160, p=2 \times 10^{-8}$ and circulating BCAA GRS with HOMA-IR, $\beta=-0.021, p=0.4$ ).

The insulin resistance GRS increased fasting insulin levels by $0.037 \mathrm{SD} /$ allele $\left([95 \% \mathrm{CI} 0.15,0.059] p=9 \times 10^{-4}\right)$ and HOMA-IR by $0.033 \mathrm{SD} /$ allele in the ADDITION-PRO cohort ([95\% CI 0.012, 0.055]p=0.002). The insulin resistance GRS also significantly increased fasting plasma BCAA levels ( $\beta=0.027 \mathrm{SD} /$ allele, [95\% CI 0.005, 0.048] $p=0.01$ ), suggesting a causal effect of insulin resistance on circulating BCAA levels (Fig. 1).

To estimate the causal effect of HOMA-IR on plasma BCAA levels and vice versa in ADDITION-PRO, we performed instrumental variable analyses where the GRS for HOMA-IR ( $F$-statistic 10.4) and circulating BCAA levels $(F$-statistic 4.7$)$ were used as instruments. Consistent with the observational association between HOMA-IR and plasma BCAA levels, genetically instrumented HOMA-IR increase showed a causal effect on circulating BCAA levels in the fasting state; each SD increase in HOMA-IR increased plasma BCAA levels by 0.73 SD ([95\% CI 0.26, 1.19] $p=0.002)$. By contrast, a genetically instrumented increase in fasting plasma BCAA levels did not show a significant causal effect on HOMA-IR $(\beta=-0.27 \mathrm{SD},[95 \% \mathrm{CI}-0.054,0.031] p=0.6)$ (Fig. 1). Excluding the rs9637599 SNP from the GRS for 
Fig. 1 Mendelian randomisation study. Fasting plasma amino acid levels and HOMA-IR were inverse-normally transformed and $\beta$ values (adjusted for age, sex and BMI) are expressed in units of SD of the inverse-normally transformed traits, except for the results from the MAGIC consortium, where the $\beta$ values are expressed in log transformed units [4]. ${ }^{1}$ The results shown are for the valine-increasing GRS
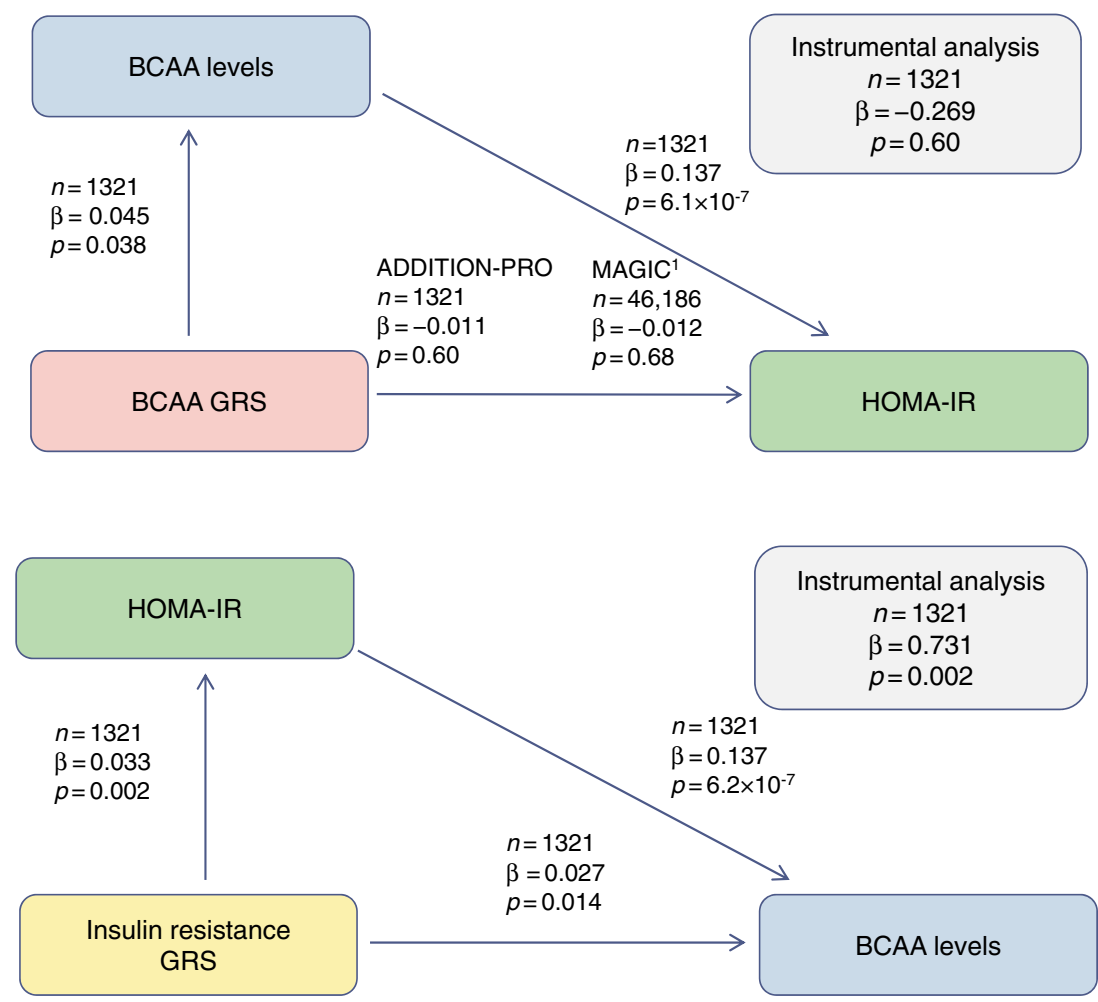

circulating BCAA levels, our results remained unchanged (data not shown).

The lack of association between the GRS for circulating BCAA levels and indices of insulin resistance could be due to the small sample size of ADDITION-PRO. Therefore, we implemented additional GRS analyses using a method that approximates the average effect of variants increasing circulating BCAA levels on insulin resistance, and that of insulin-resistance-increasing variants on circulating BCAA levels, in publicly accessible GWAS meta-analysis results [5, 14] (ESM Table 4). In these analyses, we examined GRSs for the three circulating BCAAs (isoleucine, leucine,

Table 1 Association of the BCAA GRS with fasting glycaemic traits and plasma BCAA levels in the ADDITION-PRO cohort

\begin{tabular}{lll}
\hline Variable & $\beta(95 \% \mathrm{CI})$ & $p$ \\
\hline Fasting plasma isoleucine & $0.027(-0.015,0.069)$ & 0.202 \\
Fasting plasma leucine & $0.057(0.016,0.098)$ & 0.007 \\
Fasting plasma valine & $0.048(0.003,0.093)$ & 0.036 \\
Fasting total plasma BCAA & $0.045(0.002,0.087)$ & 0.038 \\
Fasting plasma glucose & $-0.016(-0.064,0.032)$ & 0.502 \\
Fasting serum insulin & $-0.011(-0.053,0.032)$ & 0.615 \\
HOMA-IR & $-0.011(-0.054,0.031)$ & 0.602 \\
\hline
\end{tabular}

$\beta$ and $95 \%$ CI are expressed in units of SD per BCAA-increasing allele and were calculated from multiple linear regressions adjusted for age, sex and $\mathrm{BMI}$ valine) separately rather than combined, because of the lack of availability of GWAS results for combined levels of circulating BCAAs. Despite large sample size $(n=46,186)$, isoleucine-, leucine- and valine-increasing GRSs (rs10211524, rs9637599) were not associated with HOMA-IR $(\beta-0.024$, [95\% CI $-0.132,0.084] p=0.7$, $(\beta-0.019,[95 \% \mathrm{CI}-0.097,0.059] p=0.6)$ and $\beta-0.012$, $[95 \% \mathrm{CI}-0.069,0.045] p=0.7$, respectively). However, on the contrary, the HOMA-IR-increasing GRS was significantly associated with increased isoleucine $(\beta 1.22$, [95\% CI 0.71 , $\left.1.73] p=4 \times 10^{-6}, n=24,775\right)$, leucine $(\beta 0.96$, [95\% CI 0.45 , 1.47] $\left.p=3 \times 10^{-4}, n=24,728\right)$ and valine $(\beta 0.67,[95 \% \mathrm{CI}$ $0.16,1.18] p=0.01, n=24,900)$ levels.

\section{Discussion}

Using a GRS for circulating BCAA levels as an instrumental variable, we found no genetic evidence of a causal effect of circulating BCAA levels on insulin resistance in ADDITION-PRO or in MAGIC GWAS meta-analysis results. By contrast, our analyses using an insulin resistance GRS indicate that insulin resistance has a causal effect on plasma BCAA levels.

Increased plasma BCAA levels are associated with insulin resistance, but the role of circulating BCAAs as a potential causal factor in insulin resistance is unclear [2, 12]. In rats, administration of a mixture of a high fat diet and BCAAs is 
accompanied by chronic activation of mammalian target of rapamycin complex 1 (mTORC), p70-S6 kinase 1 and c-Jun $\mathrm{N}$-terminal kinase (JNK), and phosphorylation of IRS1 (Ser307), which contribute to the development of insulin resistance and diabetes [20]. A recent study in mice suggested that elevated BCAA levels could cause insulin resistance by promoting lipid accumulation in the muscle [3]. However, other studies suggest that elevated BCAA levels are just a marker of impaired insulin action and not a causative factor in the development of insulin resistance [2]. Our results from the Mendelian randomisation are in accordance with this hypothesis, indicating that circulating BCAAs have no causal effect on insulin resistance.

Insulin is the chief regulator of amino acid metabolism and changes in fasting BCAA levels may be regulated by the effect of insulin on the rate of appearance and clearance of BCAA together with a decreased activity of catabolic enzymes [2]. Loss-of-function mutations in PPM1K in humans [21], and disruption of key BCAA metabolism in obese mice and Zucker rats, exhibit a reduced expression of the mitochondrial isoform of branched chain-amino-acid transaminase (BCAT2; which catalyses the first and reversible step in BCAA catabolism), and mitochondrial branched chain $\alpha$-keto acid dehydrogenase (BCKD) complex E1- $\alpha$ (which catalyses the rate-controlling and the first irreversible step), leading to increased plasma BCAA levels [22, 23]. A decreased BCAA metabolism in fat tissue may contribute to higher BCAA levels in individuals with insulin-resistant obesity [2, 22-24]. Intriguingly, a recent study also showed that the gut microbiota can contribute to elevated BCAA levels in insulin-resistant states [25].

Mendelian randomisation studies exploit the fact that genotypes are randomly assigned at meiosis in order to study causal relationships between traits without being affected by confounding or reverse causality [19]. The GRS for circulating BCAA levels and HOMA-IR did not show an association with any key covariates in ADDITION-PRO (ESM Table 5). While we attempted to maximise the specificity of the genetic instruments, we cannot fully exclude the possibility of residual pleiotropy, i.e. that the selected genetic variants act not only on circulating BCAA levels or insulin resistance, but also on other metabolic pathways independent of their influence on BCAA levels or insulin resistance. Furthermore, while we were able to study the causal relationship between circulating BCAAs and insulin resistance as such, we were not able to study more detailed biological mechanisms that may mediate the causal link between the two.

In conclusion, our results do not support the hypothesis that plasma BCAA levels have a causal effect on insulin resistance, but indicate that insulin resistance drives higher circulating BCAA levels.
Data availability The genome-wide meta-analysis summary results for HOMA-IR and BCAA are found in (www.magicinvestigators.org/ downloads) and (www.computationalmedicine.fi/data\#NMR_GWAS), respectively. Other relevant data for the present study are shown in the paper and in the ESM. If you wish to see additional data, the authors confirm that, for approved reasons, some access restrictions apply to the data underlying the findings. Data is available at the Novo Nordisk Foundation Center for Basic Metabolic Research, section of Metabolic Genetics whose authors may be contacted via torben.hansen@sund.ku.dk.

Funding This study was funded by an unrestricted grant from the European Foundation for the Study of Diabetes/Pfizer for Research into Cardiovascular Disease Risk Reduction in Patients with Diabetes (74550801), the Danish Council for Strategic Research, internal research and equipment funds from Steno Diabetes Center and supported by research grants from the Novo Nordisk Foundation. The Novo Nordisk Foundation Center for Basic Metabolic Research is an independent Research Center at the University of Copenhagen partially funded by an unrestricted donation from the Novo Nordisk Foundation (www.metabol.ku.dk). YM is funded by the Danish Diabetes Academy. AJ is supported by the Danish Council for Independent Research, European Union, FP7, Marie Curie Actions, IEF and Lundbeck Foundation. The study sponsors were not involved in the design of the study; the collection, analysis, and interpretation of data; writing the report; or the decision to submit the report for publication.

Duality of interest $\mathrm{OP}$ and $\mathrm{TH}$ hold personal shares in Novo Nordisk $\mathrm{A} / \mathrm{S}$; the remaining authors declare no potential conflict of interest relevant to this article.

Contribution statement YM designed the study, analysed and researched data, and wrote the manuscript. AJ contributed NMR data, and reviewed and edited the manuscript. CTH performed imputation and quality control of the genotype data, and reviewed and edited the manuscript. KHA contributed to analysis and interpretation of data, reviewed and edited the manuscript, and contributed to discussion. DRW and MEJ designed the cohort, and reviewed and edited the manuscript. NG contributed to analysis and interpretation of data, and reviewed and edited the manuscript. OP contributed to analysis and interpretation of data, reviewed and edited the manuscript, and contributed to discussion. TOK designed the study, researched data, reviewed, wrote and edited the manuscript and contributed to discussion. TH designed the study, reviewed and edited the manuscript and contributed to discussion. All the authors contributed to the design of the study, interpretation of the data, critical revision of the article, and approved the final draft of the manuscript. TH is the guarantor of this study.

\section{References}

1. DeFronzo RA, Tripathy D (2009) Skeletal muscle insulin resistance is the primary defect in type 2 diabetes. Diabetes Care 32(Suppl 2): S157-S163

2. Lynch CJ, Adams SH (2014) Branched-chain amino acids in metabolic signalling and insulin resistance. Nat Rev Endocrinol 10: 723-736

3. Jang C, Oh SF, Wada S et al (2016) A branched-chain amino acid metabolite drives vascular fatty acid transport and causes insulin resistance. Nat Med 22:421-426

4. Scott RA, Lagou V, Welch RP et al (2012) Large-scale association analyses identify new loci influencing glycemic traits and provide insight into the underlying biological pathways. Nat Genet 44:9911005 
5. Kettunen J, Demirkan A, Wurtz P et al (2016) Genome-wide study for circulating metabolites identifies 62 loci and reveals novel systemic effects of LPA. Nat Commun 7:11122

6. Scott RA, Fall T, Pasko D et al (2014) Common genetic variants highlight the role of insulin resistance and body fat distribution in type 2 diabetes, independent of obesity. Diabetes 63:4378-4387

7. Wynn RM, Li J, Brautigam CA, Chuang JL, Chuang DT (2012) Structural and biochemical characterization of human mitochondrial branched-chain $\alpha$-ketoacid dehydrogenase phosphatase. J Biol Chem 287:9178-9192

8. Johansen NB, Hansen AL, Jensen TM et al (2012) Protocol for ADDITION-PRO: a longitudinal cohort study of the cardiovascular experience of individuals at high risk for diabetes recruited from Danish primary care. BMC Public Health 12:1078

9. Lauritzen T, Griffin S, Borch-Johnsen K et al (2000) The ADDITION study: proposed trial of the cost-effectiveness of an intensive multifactorial intervention on morbidity and mortality among people with Type 2 diabetes detected by screening. Int $\mathrm{J}$ Obes Relat Metab Disord 24(Suppl 3):S6-S11

10. Hansen AL, Carstensen B, Helge JW et al (2013) Combined heart rate- and accelerometer-assessed physical activity energy expenditure and associations with glucose homeostasis markers in a population at high risk of developing diabetes: the ADDITION-PRO study. Diabetes Care 36:3062-3069

11. Turner S, Armstrong LL, Bradford Y et al (2011) Quality control procedures for genome-wide association studies. Curr Protoc Hum Genet Chapter 1: Unit1.19, doi:10.1002/0471142905.hg0119s68

12. Howie BN, Donnelly P, Marchini J (2009) A flexible and accurate genotype imputation method for the next generation of genomewide association studies. PLoS Genet 5:e1000529

13. Marchini J, Howie B (2010) Genotype imputation for genome-wide association studies. Nat Rev Genet 11:499-511

14. Dupuis J, Langenberg C, Prokopenko I et al (2010) New genetic loci implicated in fasting glucose homeostasis and their impact on type 2 diabetes risk. Nat Genet 42:105-116
15. Locke AE, Kahali B, Berndt SI et al (2015) Genetic studies of body mass index yield new insights for obesity biology. Nature 518:197206

16. Berndt SI, Gustafsson S, Magi R et al (2013) Genome-wide metaanalysis identifies 11 new loci for anthropometric traits and provides insights into genetic architecture. Nat Genet 45:501-512

17. Dastani Z, Hivert MF, Timpson N et al (2012) Novel loci for adiponectin levels and their influence on type 2 diabetes and metabolic traits: a multi-ethnic meta-analysis of 45,891 individuals. PLoS Genet 8:e1002607

18. International Consortium for Blood Pressure Genome-Wide Association Studies, Ehret GB, Munroe PB et al (2011) Genetic variants in novel pathways influence blood pressure and cardiovascular disease risk. Nature 478:103-109

19. Baiocchi M, Cheng J, Small DS (2014) Instrumental variable methods for causal inference. Stat Med 33:2297-2340

20. Newgard CB, An J, Bain JR et al (2009) A branched-chain amino acid-related metabolic signature that differentiates obese and lean humans and contributes to insulin resistance. Cell Metab 9:311-326

21. Oyarzabal A, Martinez-Pardo M, Merinero B et al (2013) A novel regulatory defect in the branched-chain $\alpha$-keto acid dehydrogenase complex due to a mutation in the PPM1K gene causes a mild variant phenotype of maple syrup urine disease. Hum Mutat 34:355-362

22. She P, Van Horn C, Reid T, Hutson SM, Cooney RN, Lynch CJ (2007) Obesity-related elevations in plasma leucine are associated with alterations in enzymes involved in branched-chain amino acid metabolism. Am J Phys Endocrinol Metab 293:E1552-E1563

23. She P, Olson KC, Kadota Y et al (2013) Leucine and protein metabolism in obese Zucker rats. PLoS One 8:e59443

24. Lackey DE, Lynch CJ, Olson KC et al (2013) Regulation of adipose branched-chain amino acid catabolism enzyme expression and cross-adipose amino acid flux in human obesity. Am J Physiol Endocrinol Metab 304:E1175-E1187

25. Pedersen HK, Gudmundsdottir V, Nielsen HB et al (2016) Human gut microbes impact host serum metabolome and insulin sensitivity. Nature 535:376-381 